\title{
Extensive atrial wall resection in a patient with symptomatic idiopathic right atrial enlargement
}

 \\ Guillermo Touyá, MD, and Diego Abdala, MD, ${ }^{\mathrm{b}}$ Montevideo, Uruguay
}

I

diopathic right atrial enlargement is an uncommon cardiac anomaly, almost always asymptomatic and of unknown etiology. ${ }^{1,2}$ Although frequently considered a relatively benign disorder, ${ }^{3,4}$ this lesion may be associated with atrial arrhythmias, severe tricuspid regurgitation, thrombus formation, and even sudden death. ${ }^{1-4}$ We report the case of a 45 -year-old man who underwent surgical correction of symptomatic idiopathic right atrial enlargement.

\section{Clinical Summary}

A 45-year old-man with idiopathic right atrial enlargement was seen with chest discomfort, moderate dyspnea, and atrial fibrillation. At admission, pericardial effusion was suspected because of a markedly enlarged cardiac silhouette on chest radiography (Figure 1,A). Echocardiography performed during the patient's evaluation revealed an extremely enlarged right atrium, severe tricuspid insufficiency from annular dilatation, and a large intra-atrial thrombus $(10 \mathrm{~cm})$. Both ventricles were displaced leftward, with normal systolic function. Ebstein anomaly was excluded because of normal tricuspid valve attachment. The diagnosis of idiopathic right atrial enlargement was based on the findings of a giant right atrium, leading to tricuspid annular dilatation, with an otherwise normal heart.

Because of his symptoms, severe tricuspid regurgitation, and high risk of massive pulmonary embolism, the patient was referred to our unit for surgical treatment. At the operation, access was established through a median sternotomy. The enlarged, paper-thin right atrial wall filled the entire pericardial cavity. With normothermic cardiopulmonary bypass and without aortic occlusion, a thrombus large enough to fill the surgeon's hand completely was removed from the cavity.

The tricuspid annulus was dilated, but the rest of the apparatus appeared normal. Extensive right atrial wall resection (Figure 2)

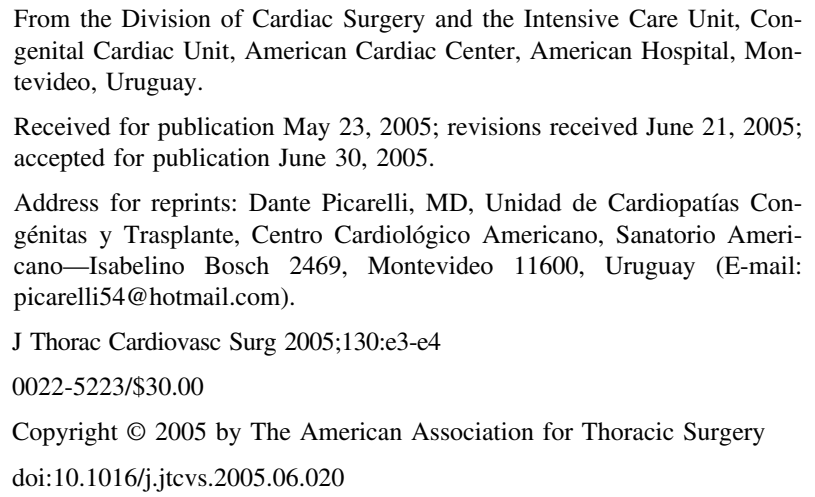

and De Vega annuloplasty were performed. The patient made an uneventful recovery, despite a pericardial effusion successfully managed without

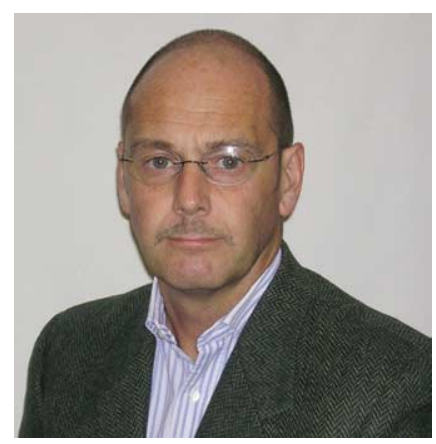

Dr Picarelli surgical drainage.

Postoperative chest radiography showed a nearly normal cardiac silhouette (Figure 1,B). Postrepair echocardiography revealed a markedly reduction of the right atrium size, without residual tricuspid insufficiency or intra-atrial thrombus.

Macroscopic right atrial wall examination demonstrated an extreme wall thinning; the histologic study confirmed the reduction of muscular tissue and lipomatous degeneration. Anticoagulation was required for persistent atrial fibrillation. Twelve months after surgery, the patient is free of symptoms and in good condition.

\section{Discussion}

Idiopathic right atrial enlargement is a rare abnormality that must be distinguished from more common structural cardiac lesions leading to right atrial dilation, such as Ebstein anomaly and other lesions of the right ventricle, tricuspid valve, or pulmonary valve. ${ }^{2-4}$ Frequently asymptomatic, in most patients it is discovered incidentally after recognition of an enlarged cardiac silhouette during routine chest radiography. ${ }^{1-3}$ Its etiology remains unknown, and controversy persists about the origin of this condition. ${ }^{1,2}$

Like others, ${ }^{4}$ we found a reduction of muscular fibers and lipomatous degeneration of the right atrial wall. Differentiation from other causes of enlarged cardiac silhouette is important for proper medical and surgical management. An accurate diagnosis avoids unnecessary and potentially dangerous procedures, such as pericardiocentesis or surgical exploration. Echocardiography was in our case the technique of choice to establish the diagnosis, excluding other causes of right atrial dilatation and enlarged cardiac silhouette.

Because of the small number of patients with symptoms, little is known about the long-term prognosis of this condition, making hazardous the decision of the best approach to symptom-free patients. ${ }^{1-4}$ Previous reports ${ }^{3}$ have indicated the relatively benignity of this disorder, which is usually without symptoms. However, severe complications and even sudden death have been reported. ${ }^{1,2,4}$ Symptoms have been attributed to atrial arrhythmias, ventricular compression, and tricuspid regurgitation. ${ }^{3}$ In addition, thrombus formation increases the risk of embolic events. ${ }^{1,3,4}$

Although some patients without symptoms have been treated surgically, ${ }^{1}$ this anomaly can be managed medically for long periods, ${ }^{3}$ with surgery being reserved for patients with symptoms 

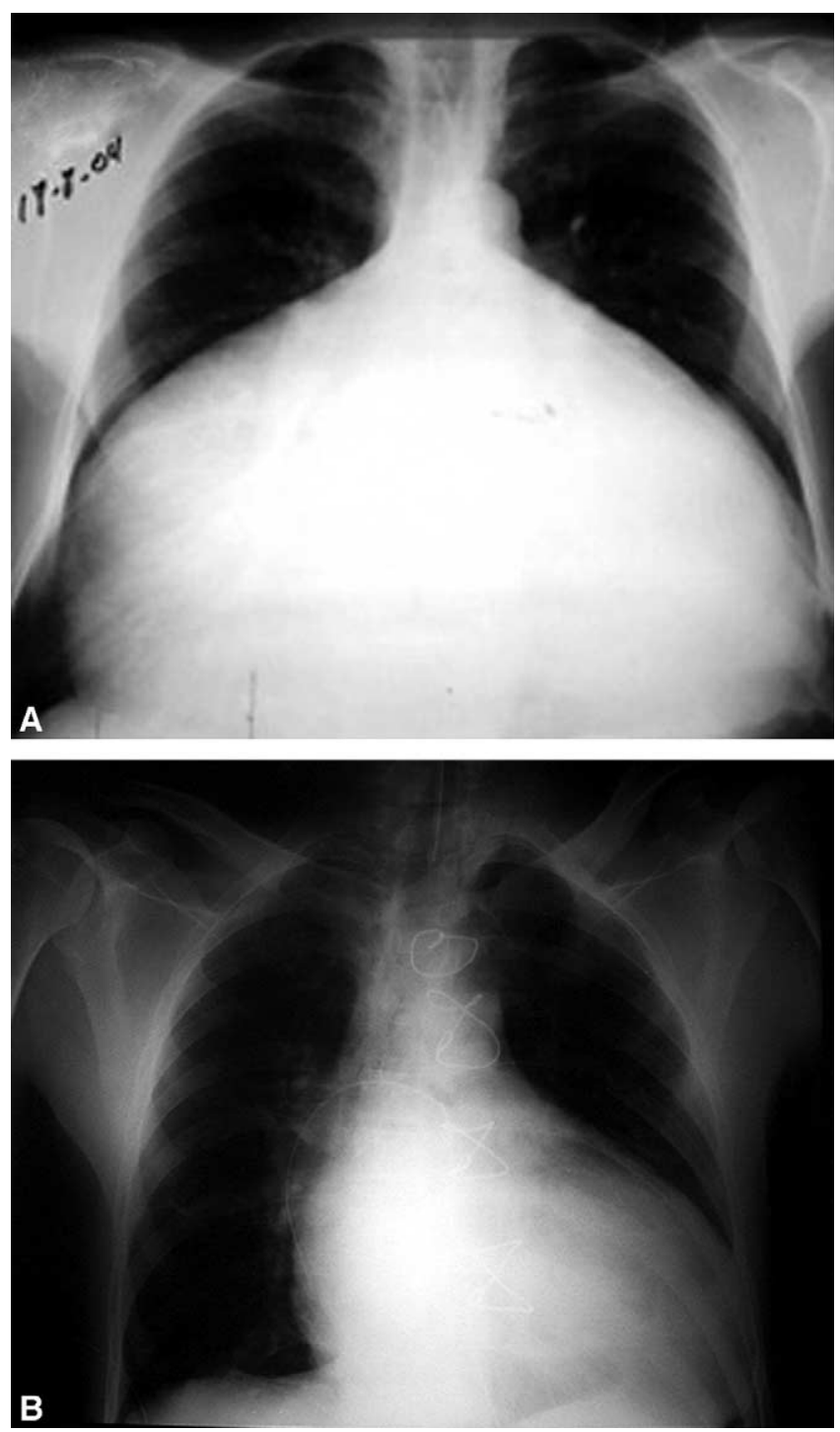

Figure 1. A, Markedly enlarged cardiac silhouette is seen on preoperative chest radiography. B, Nearly normal cardiac silhouette is seen on postoperative chest radiography.

such as uncontrolled arrhythmias, severe tricuspid regurgitation, or high-risk thromboembolic complications. ${ }^{2,4}$ Our extensive right atrial resection included the free wall of the atrium. Because of the structural atrial wall anomaly, every effort was made to leave

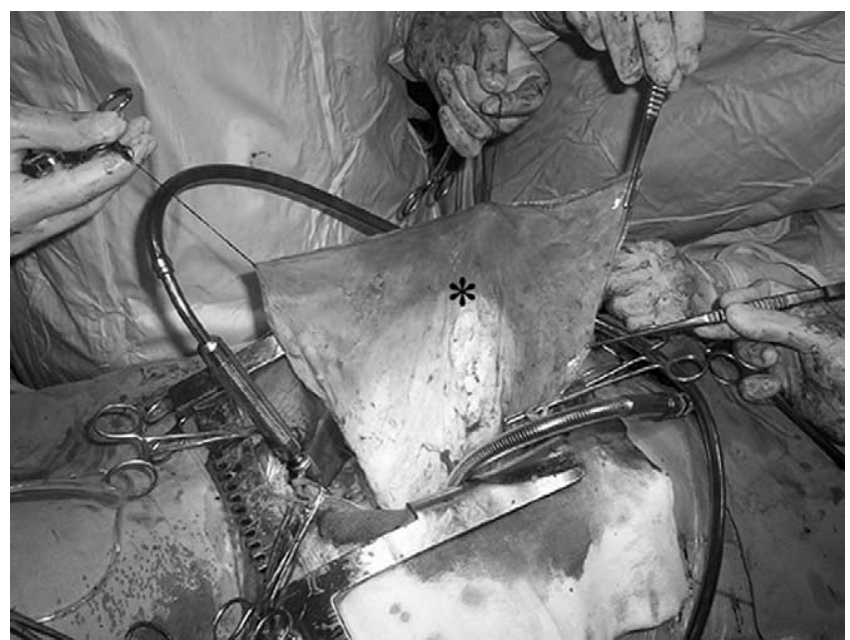

Figure 2. Partial resected right "paper thin" atrial wall.

enough atrial tissue to perform a tension-free suture line between both paper-thin edges. In our patient, atrial size reduction and tricuspid annuloplasty improved hemodynamics and clinical condition. In contrast with others' reports, ${ }^{1,2,4}$ however, we were unsuccessful in abolishing the arrhythmogenic substrate of atrial fibrillation. Surgical cryoablation was not attempted, because its effectiveness in this condition remains controversial. ${ }^{1}$

We believe that the successful outcome of this case validates the surgical treatment of patients with symptomatic idiopathic right atrial enlargement.

We thank Christian Kreutzer MD, for successful criticism of the manuscript.

\section{References}

1. Chatrath R, Turek O, Quivers ES, Driscoll DJ, Edwards WD, Danielson GK. Asymptomatic giant right atrial aneurysm. Tex Heart Inst. 2001; 28:301-3.

2. Rutledge J, Robertson MA, Kantoch M, Dyck J. Idiopathic dilation of the right atrium: case report and survey of the literature. Can J Cardiol. 1997; 13:855-7.

3. Kurz DJ, Oechslin EN, Kobza R, Jenni R. Idiopathic enlargement of the right atrium: 23 year follow up of a familial cluster and their unaffected relatives. Heart. 2004;90:1310-4.

4. Binder TM, Rosenhek R, Frank H, Gwechenberger M, Maurer G, Baumgartner $\mathrm{H}$. Congenital malformations of the right atrium and the coronary sinus: an analysis based on 103 cases reported in the literature and two additional cases. Chest. 2000;117:1740-8. 